\title{
Fatigue damage accumulation in CFRP
}

\author{
A. Plumtree ${ }^{1} \&$ K. Cain ${ }^{2}$ \\ ${ }^{I}$ Department of Mechanical and Mechatronics Engineering, \\ University of Waterloo, Canada \\ ${ }^{2}$ School of Engineering Technology, Conestoga College, Canada
}

\begin{abstract}
A cyclic bending study using a stress ratio of 0.1 was conducted on unidirectional $45^{\circ} \mathrm{CFRP}$ with a $60 \%$ nominal fibre volume fraction. This allowed the continuous matrix damage evolution to be followed without the influence of delamination. Damage was monitored by changes in the fatigue modulus, increase in crack density and amount of permanent bending. It was found that their effects were complementary and that, in general, damage evolved in the same way as that observed in unidirectional tension-tension cyclic tests. All three monitoring techniques showed that damage accumulation was cycle dependent. Damage may be divided into two main stages, with rapid growth at the onset of cycling to about $10-20 \%$ life (Stage I), after which the damage increases slowly, but steadily, to failure (Stage II).
\end{abstract}

Keywords: CFRP, polymer matrix composites, fatigue damage accumulation, cyclic bending, two stage damage.

\section{Introduction}

Fatigue damage in polymer matrix composites occurs in several forms including matrix cracking, fibre fracture and buckling, debonding at the matrix/fibre interface and delamination between plies. Initial cracking occurs preferentially in the relatively weak matrix. Since there are many stress concentration sites in the matrix adjacent to the fibres, matrix damage is a process of initiation and gradual coalescence. Because of continual crack initiation and propagation, matrix cracking is regarded as progressive damage, as opposed to non progressive damage of the fibres. 
It has been observed that fatigue damage caused by unidirectional cycling composites occurs in two dominant stages involving an initially high but decreasing damage rate followed by a slowly increasing rate [1,2]. The first consists of homogeneous non-interactive cracking restricted to individual plies. Damage develops at a high but decreasing rate due to the exhaustion of damage sites which relax the internal stress created by the applied load. The transition from the first to the second stage occurs when a balance between crack density and applied load is established (Characteristic Damage State). Generally, the specimen will exhibit a well-defined crack pattern. The second stage is characterized by the localization of damage in zones of increasing crack interaction by delamination and fibre fracture which lead to an overall steady state increase in damage until just before fracture takes place. The proportion and amount of damage occurring during each stage depends upon the configuration of the composite and the imposed stress level.

The intent of the current study is to show that the two stage model is applicable to cyclic bending of a unidirectional CFRP composite while concentrating on matrix damage accumulation by reducing the influence of other damage mechanisms.

\section{Procedure}

The composite studied was constructed of $60 \%$ volume fraction HTA carbon fibre reinforced with epoxy 6376. Specimens were machined from a unidirectional 8 ply plate at an angle of $45^{\circ}$ to the loading axis. Their size was approximately $90 \mathrm{~mm}$ long, $16 \mathrm{~mm}$ wide and $1 \mathrm{~mm}$ thick. The edges were sanded and polished with fine emery paper (600grit).

A servo-hydraulic test rig was used to conduct three-point bending fatigue tests. All the tests were performed at room temperature and a frequency of $4 \mathrm{~Hz}$ with a sinusoidal waveform and a stress ratio $(\mathrm{R}=$ minimum stress/maximum stress) of 0.1 . Using a $22.2 \mathrm{kN}$ load cell and a strain gauge mounted on the tensile surface of each specimen, the stress and strain data were recorded.

Some tests were stopped periodically to measure the amount of permanent bending and the corresponding number or cracks. For the crack counting procedure using an optical microscope, the tensile surface was examined at a magnification of $500 \mathrm{x}$ on polished areas of approximately $16000 \mu^{2}$. The fracture surfaces were examined in a JEOL scanning electron microscope.

\section{Results}

\subsection{Static tests}

The tensile strength and modulus of elasticity were determined using the same test rig and procedure as that for the fatigue tests. The average values of three tests were - Tensile Strength: 261.4 MPa and Modulus of Elasticity: 16.4 GPa. 


\subsection{Fatigue tests}

The cyclic stress $(\sigma)$ - life $\left(\mathrm{N}_{\mathrm{F}}\right)$ curve for $\mathrm{R}=0.1$ was determined and expressed by $\sigma=270-31.25 \log \mathrm{N}_{\mathrm{F}}$

Data from the strain gauges allowed changes in stiffness to be recorded. The decrease of the material stiffness with cycles was expressed in terms of the fatigue damage parameter, $\mathrm{D}_{\mathrm{F}}$, by:

$$
\mathrm{D}_{\mathrm{F}}=1-E_{1}^{N} / \mathrm{E}_{\mathrm{o}}
$$

where $E_{1}^{N}$ is the fatigue modulus after $\mathrm{N}_{1}$ cycles and $\mathrm{E}_{\mathrm{o}}$ is the initial modulus measured during the first cycle. Figure 1 shows the development of the fatigue damage parameter for different stress levels. In general, the parameter is cycle dominant, rather than stress. The curves can be divided into two significant parts. The first $10-20 \%$ of life is defined by a rapid increase in damage. At the changeover to Stage II for $80-90 \%$ life the slope decreases, although the increase in damage parameter is still significant.

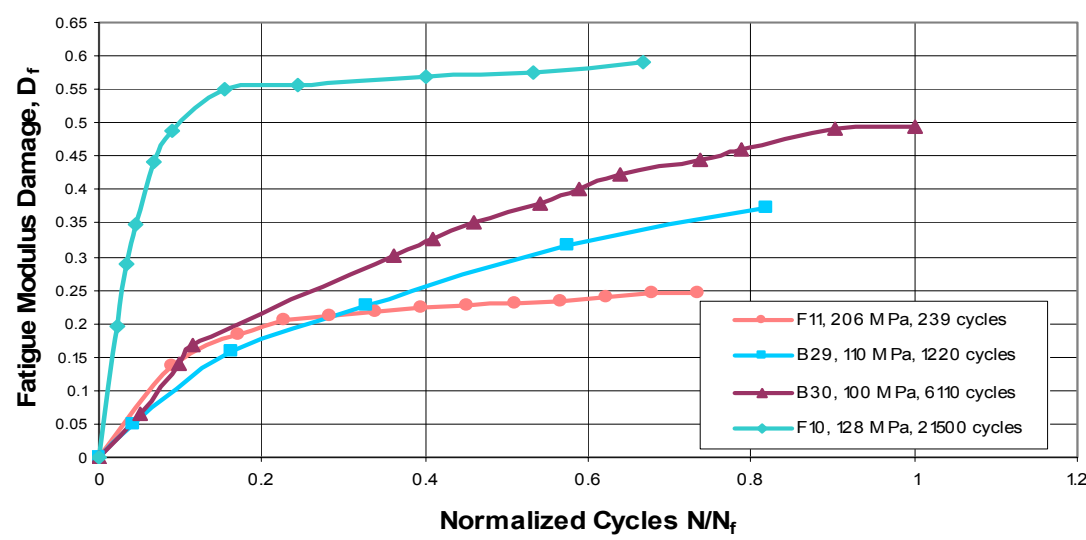

Figure 1: $\quad$ Fatigue damage parameter for a $[45]_{8}$ HTA/6376 composite under three-point cyclic bending at a frequency of $4 \mathrm{~Hz}$ and $\mathrm{R}=$ 0.1 .

\subsection{Crack density}

Crack density measurements were determined by monitoring the number of cracks on the tensile surface of the specimens. Crack density is a bulk material characteristic since the final fracture site was not observed in the areas viewed under microscope. Figure 2 shows the evolution of the number of cracks with increasing normalized cycles for a typical three-point bending specimen. It can be seen that the initial number of cracks increased quickly with increasing number of cycles, and that the rate of increase dropped dramatically after about $20 \%$ life. Cracks accumulated in a consistent manner during this second stage slowly at the lower stresses, hence lower growth rates. However, during the first stage the crack distribution was established, which determined the location of the incipient fatal crack. 
For four-point cyclic bending, the number of cracks also increased in those specimens experiencing higher stress levels, as shown in Table 1 [3]. The same trends have been observed in unidirectional cyclic tension samples, $\mathrm{R}=0.1[4,5]$.

Crack accumulation, shown in Figure 2 can be represented by the two stage model related to the development damage [2]. An initial rapid increase in matrix cracking takes place for the first $20 \%$ life (Stage I), followed by slower accumulation (Stage II) due to crack coalescence and fibre fracture [4]. Stage I cracking also corresponds to the initial rapid increase in the fatigue damage parameter, which is greater for longer lives. As the initial crack density slows significantly, a Characteristic Damage State is reached signifying the end of Stage I. This indicates a balance having been established between the applied load and crack density. Thereafter, the number of cracks and their lengths increase slowly over the remainder of life (Stage II) which is reflected by a gradual increase in the fatigue damage parameter.

Examination of the fracture surfaces under the scanning electron microscope showed such characteristic features as matrix hackles (A) and broken fibres (B), as seen in Figure 3. More significantly, the specimens displayed debris (C) and smooth fibres with no matrix material adhering to them (D). This was more evident after large numbers of cycles, indicating that the fibre/matrix interface had broken down, forming debris consisting of matrix particles [6].

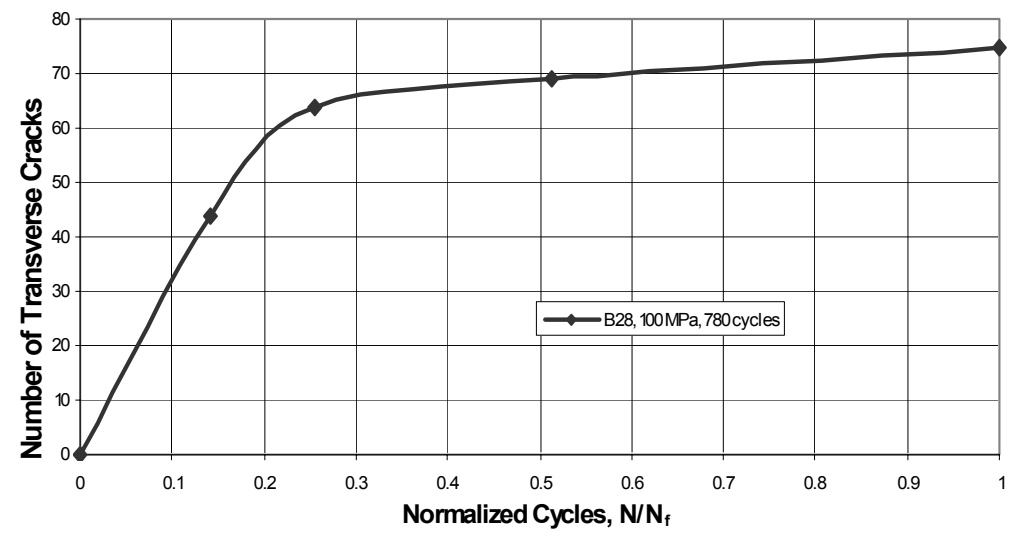

Figure 2: $\quad$ Development of transverse cracks in $16,000 \mu \mathrm{m}^{2}$ on the tensile side of $45^{\circ}$ CFRP specimen tested in 3-pt cyclic bending, $\mathrm{R}=0.1$.

Table 1: $\quad$ Crack densities: $10^{\circ}$ CFRP 4-pt cyclic bending, $\mathrm{R}=0.1$ [3].

\begin{tabular}{|l|l|l|c|c|c|}
\hline Specimen & $\begin{array}{l}\text { Strain } \\
\times 10^{-3}\end{array}$ & $\begin{array}{c}\text { Stress } \\
(\mathrm{MPa})\end{array}$ & $\begin{array}{c}\text { Cycles } \\
\text { to } \\
\text { Failure }\end{array}$ & $\begin{array}{c}\text { Total } \\
\text { Cracks } / \mathrm{mm}^{2}\end{array}$ & $\begin{array}{c}\text { Matrix } \\
\text { Cracks } / \mathrm{mm}^{2}\end{array}$ \\
\hline L1 & 7.29 & 66 & 1986 & 1007 & 903 \\
\hline L2 & 5.75 & 52 & 534718 & 4132 & 1558 \\
\hline
\end{tabular}




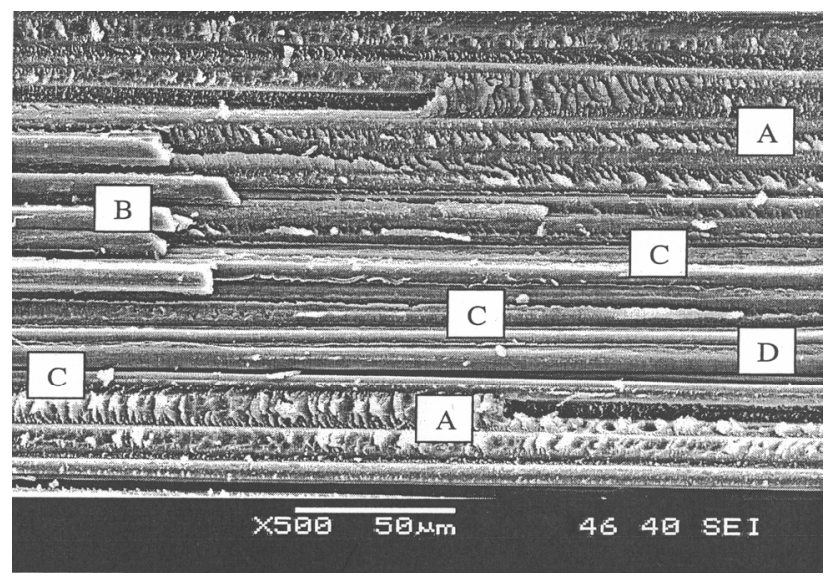

Figure 3: $\quad$ Fracture surface: A hackles, B broken fibres, C debris, D smooth fibres.

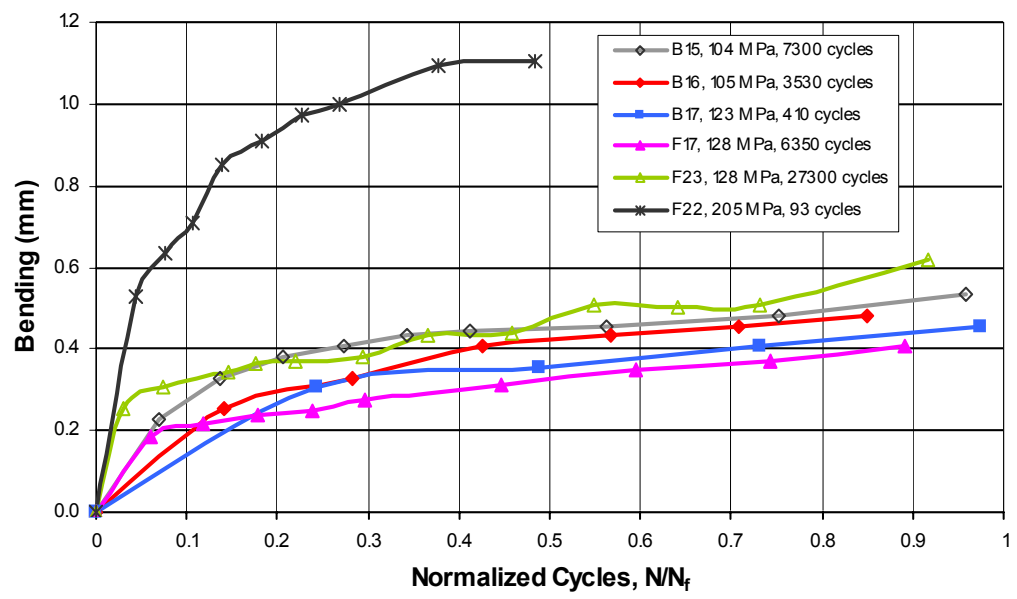

Figure 4: $\quad$ Permanent bending in $45^{\circ}$ CFRP tested in cyclic 3-pt bending at $\mathrm{R}=0.1$. Normalized cycles abscissa.

\subsection{Bending}

Permanent bending is the curvature of the specimen, resulting from cracking and visco-elastic effects. Companion specimens tested to the same stress level in monotonic bending showed immediate relaxation upon unloading, whereas bending was permanent for fatigue specimens [7]. Figure 4 shows the bending of specimens cycled at different stress levels. Permanent deflection displayed a two-stage response. The majority of permanent bending took place within the first $10-20 \%$ of life (Stage I) and the rate of deflection then dropped significantly (Stage II). Larger deflections occurred at higher cyclic stress levels. 


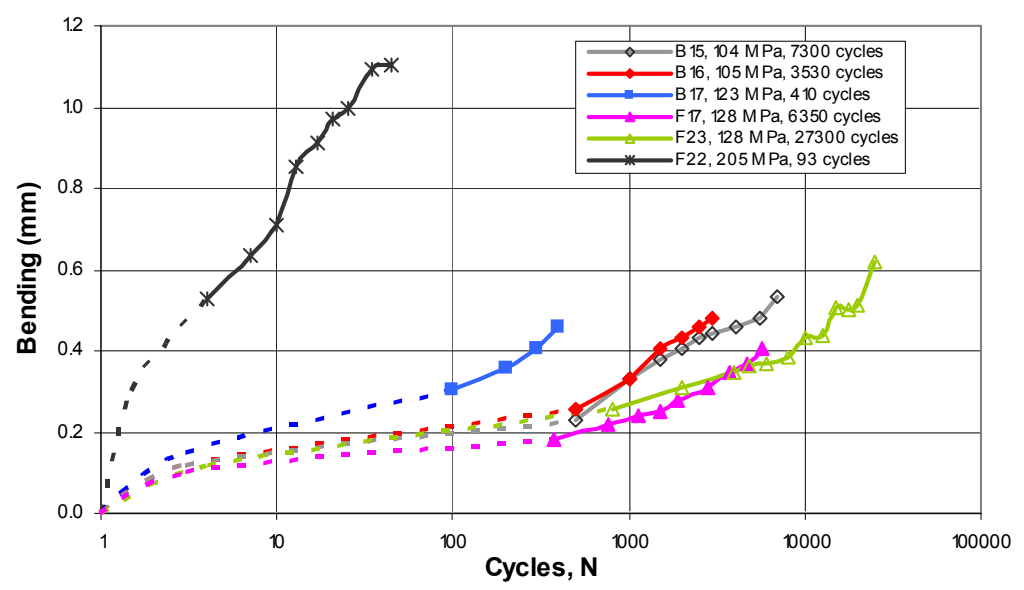

Figure 5: $\quad$ Permanent bending in $45^{\circ}$ CFRP tested in cyclic 3-pt bending at $\mathrm{R}=0.1$. Logarithmic cycles abscissa.

Figure 5 shows the same data as Figure 4, but with logarithmic number of cycles as the abscissa. Now the curves resemble creep behaviour. The secondary creep rate increased with increase in stress. However, the amount of permanent bending is a combination is of local and general bulk deflection. In the case of the higher stress level, the larger deflection indicated a higher degree of viscoelasticity and poorer distribution of cracks, with a large number confined to a small region directly beneath the point of loading. In this case the small bend signified a large amount of bending over a confined region.

\section{Discussion}

The results show that, as with unidirectional cycling, cyclic bending damage evolution can be divided into two main stages. Rapid growth occurs at the onset of loading (Stage I) and a levelling off occurs after about 10-20\% life (Stage II). The transition from Stage I to Stage II indicates a change in damage mechanism. During Stage II, the three monitoring methods show a more gradual increase in the amount of damage. However, the effect of the fatal crack was not seen. It is estimated that the fatal crack only becomes evident after approximately $90-99 \%$ life [6].

During the first $20 \%$ life, the number of transverse cracks increased very rapidly, but the initiation rate decelerated. It appears that the transverse cracks were responsible for the large decrease in stiffness over the first $20 \%$ life and partly for the development of permanent bending over the whole life. After the first $20 \%$ life, other fracture mechanisms such as fibre/matrix crack formation, coalescence and fibre breakage become prominent in this second stage. 
The changes in crack density were reflected by similar changes in the fatigue modulus. This involves a strain shift in the hysteresis loop as well as a tilt. In the present work the fatigue modulus was dominated by the former, indicating that cyclic creep or ratchetting increased with cycles. Similar behaviour has been observed in non-crimped glass-epoxy composites [8] where fatigue modulus and crack density showed the same behaviour. A large amount of damage in the form of cracks developed during the first cycle. The number of shear cracks increased quickly in the first $20 \%$ life (Stage I), then more slowly to failure (Stage II). As in the present case, crack density was cycle, not stress, dependent.

Permanent bending is the consequence of several damage mechanisms such as viscoelastic behaviour, non homogeneous deformation and debris inclusion in cracks. The first two increase with stress and the last with cycles. Viscoelastic behaviour of the polymer matrix leads to greater deformation and permanent bending at the higher stresses, as observed. Also, these high stresses result in a poor distribution of cracks. Such concentrated damage causes a small bend radius to form, producing a large deflection. Consequently, assessing damage by the amount of permanent bending reveals a poor general crack distribution along the specimen which causes early failure. Permanent bending in the cycled specimens can also be attributed to the accumulation of matrix debris in the cracks. During cycling, the matrix particles collect in the open cracks on the tensile side. On unloading, these cracks cannot close completely resulting in permanent bending.

Statically loaded specimens have shown no permanent bending [7] indicating that no debris had formed to keep the crack open on the tensile side of the sample because of lack of relative motion between the fibres and matrix.

Petermann [6] showed that more debris formed after large numbers of cycles, indicating that erosion was operating at the fibre-matrix interface. The effects of debris have been examined by Ewart and Suresh [9], and Wilson and Case [10] in cyclic compression/tension tests on ceramics. They observed the occurrence and accumulation of debris between crack faces, which reduced the crack opening displacement. They concluded that this reduced the crack growth since previous test results had shown higher crack propagation rates when the debris was periodically removed.

\section{Conclusions}

In a unidirectional $45^{\circ}$ CFRP composite tested under cyclic bending, damage evolution was found to be a two stage process, similar to that observed under unidirectional tension-tension. A large amount of damage accumulated in Stage I. The transition from Stage I to Stage II, where the damage accumulated slowly, occurred after $10-20 \%$ life. Three methods were employed with each monitoring different aspects of damage. However, all three showed the same general behaviour.

Specifically:

1. The amount of cracking was cycle dominant. The number of transverse matrix cracks increased with increasing number of cycles and decreasing stress level. In long life specimens the damage was well distributed. 
2. The fatigue damage modulus was also cycle dependent. This was dominated by cyclic creep that increased with cycles.

3. The amount of permanent bending increased with cyclic stress. It is suggested that within those specimens experiencing high stresses, the damage distribution was highly localized, resulting in a small bend radius and large deflection.

4. The fatigue life can be assessed using each of the monitoring methods by determining the transition from Stages I to II, knowing that there is $80-90 \%$ life remaining. All the assessment methods monitored different characteristics of damage accumulation within the composite, although the fatigue modulus parameter had the benefit of in-situ measurements.

\section{Acknowledgements}

The authors acknowledge the Natural Sciences and Engineering Council of Canada for financial assistance. They would like to thank Martin Ostgathe and Ahmad El-Sayed for technical assistance and Marlene Dolson for typing the manuscript.

\section{References}

[1] Talreja, R., Fatigue of Composite Materials, Technomic Publishing Company Inc., Lancaster, Pa., 177, 1987.

[2] Plumtree, A., \& Shen, G., Prediction of failure damage development in unidirectional long fibre composites. Polymers and Polymer Composites, 2, pp. 83-90, 1994.

[3] Plumtree, A., \& Shi, L., Fatigue damage evolution in off-axis unidirectional CFRP, International Journal of Fatigue, 24, pp. 155-159, 2002.

[4] Cain, K.J., Glinka, G. \& Plumtree, A., Cyclic damage characterization of an off-axis unidirectional graphite bismaleimide composite, Canadian Metallurgical Quarterly, 45, pp. 433-440, 2006.

[5] Hwang, W., \& Han, K.S., Cumulative Damage Models and Multi-Stress Fatigue Life Prediction, Composite Materials: Fatigue and Fracture, ed. P.A. Lagrace, ASTM STP 1012, pp. 87-102, 1989.

[6] Petermann, J., A Contribution to Evaluate and Predict the Strength and Life Time of Angle-Ply CFRP Laminates under Static and Cyclic Loads. Ph.D. Thesis, Technical University of Hamburg-Harburg, 2004.

[7] Plumtree, A., \& Ostgathe, M., Matrix fatigue damage evolution in a longitudinal CFRP composite, Proceedings of the Eleventh International Conference on Fracture, ed. A. Carpinteri, Politecnico di Torino, Italy, Paper 3342, pp. 1-5, 2005.

[8] Plumtree, A., \& Melo, M., Cyclic damage evolution in a PMC laminate. Key Engineering Materials, 348-349, pp. 33-36, 2007. 
[9] Ewart, L., \& Suresh, S., Crack propagation in ceramics under cyclic loads, Journal of Materials Science, 22, pp. 1173-1192, 1987.

[10] Wilson, B., \& Case, E., Low cycle mechanical and thermal fatigue of ceramics and ceramic composites with consideration of debris accumulation effects. Proceedings of the American Society for Composites, pp. 931-940, 1994. 